PROCEEDINGS OF THE

AMERICAN MATHEMATICAL SOCIETY

Volume 137, Number 10, October 2009, Pages 3497-3510

S 0002-9939(09)09945-6

Article electronically published on May 28, 2009

\title{
ON NOTIONS OF HARMONICITY
}

\author{
ZHEN-QING CHEN
}

(Communicated by Edward C. Waymire)

\begin{abstract}
In this paper we address the equivalence of the analytic and probabilistic notions of harmonicity in the context of general symmetric Hunt processes on locally compact separable metric spaces. Extensions to general symmetric right processes on Lusin spaces, including infinite dimensional spaces, are mentioned at the end of this paper.
\end{abstract}

\section{INTRODUCTION}

It is known that a function $u$ being harmonic in a domain $D \subset \mathbb{R}^{n}$ can be defined or characterized by $\Delta u=0$ in $D$ in the distributional sense; that is, $u \in W_{\text {loc }}^{1,2}(D):=$ $\left\{v \in L_{\text {loc }}^{2}(D) \mid \nabla v \in L_{\text {loc }}^{2}(D)\right\}$ so that

$$
\int_{\mathbb{R}^{n}} \nabla u(x) \cdot \nabla v(x) d x=0 \quad \text { for every } v \in C_{c}^{\infty}(D) .
$$

This is equivalent to the following averaging property by running a Brownian motion $X$ : for every relatively compact subset $U$ of $D$ :

$$
u\left(X_{\tau_{U}}\right) \in L^{1}\left(\mathbf{P}_{x}\right) \quad \text { and } \quad u(x)=\mathbf{E}_{x}\left[u\left(X_{\tau_{U}}\right)\right] \quad \text { for every } x \in U .
$$

Here $\tau_{U}:=\inf \left\{t \geq 0: X_{t} \notin U\right\}$. Recently there has been interest (e.g. [2]) from several areas of mathematics in determining whether the above two notions of harmonicity remain equivalent in a more general context, such as for diffusions on fractals (see [1]) and for discontinuous processes including symmetric Lévy processes. For instance, due to their importance in theory and in applications, there has been intense interest recently in studying discontinuous processes and non-local (or integro-differential) operators by both analytical and probabilistic approaches. See, e.g., [5, 6] and the references therein. So it is important to identify the connection between the analytic and probabilistic notions of harmonic functions.

In this paper, we address the question of the equivalence of the analytic and probabilistic notions of harmonicity in the context of symmetric Hunt processes on local compact separable metric spaces. Let $X$ be an $m$-symmetric Hunt process on a locally compact separable metric space $E$ whose associated Dirichlet form $(\mathcal{E}, \mathcal{F})$ is regular on $L^{2}(E ; m)$. Let $D$ be an open subset of $E$ and $\tau_{D}$ be the first exit time from $D$ by $X$. Motivated by the example at the beginning of this section,

Received by the editors October 25, 2008, and, in revised form, February 16, 2009.

2000 Mathematics Subject Classification. Primary 60J45, 31C05; Secondary 31C25, 60J25.

Key words and phrases. Harmonic function, uniformly integrable martingale, symmetric Hunt process, Dirichlet form, Lévy system.

The research of this author is supported in part by NSF Grant DMS-0600206.

(c) 2009 Zhen-Qing Chen 
loosely speaking (see the next section for precise statements), there are two ways to define a function $u$ being harmonic in $D$ with respect to $X$ : (a) (probabilistically) $t \mapsto u\left(X_{t \wedge \tau_{D}}\right)$ is a $\mathbf{P}_{x}$-uniformly integrable martingale for quasi-every $x \in D$; (b) (analytically) $\mathcal{E}(u, g)=0$ for $g \in \mathcal{F} \cap C_{c}(D)$. We will show in Theorem 2.11 below that these two definitions are equivalent. Note that even in the Brownian motion case a function $u$ that is harmonic in $D$ is typically not in the domain $\mathcal{F}$ of the Dirichlet form. Denote by $\mathcal{F}_{\text {loc }}^{D}$ the family of functions $u$ on $E$ such that for every relatively compact open subset $D_{1}$ of $D$, there is a function $f \in \mathcal{F}$ so that $u=f m$ a.e. on $D_{1}$. To show these two definitions are equivalent, the crux of the difficulty is to

(i) appropriately extend the definition of $\mathcal{E}(u, v)$ to functions $u$ in $\mathcal{F}_{\text {loc }}^{D}$ that satisfy some minimal integrability condition when $X$ is discontinuous so that $\mathcal{E}(u, v)$ is well defined for every $v \in \mathcal{F} \cap C_{c}(D)$;

(ii) show that if $u$ is harmonic in $D$ in the probabilistic sense, then $u \in \mathcal{F}_{\text {loc }}^{D}$ and $\mathcal{E}(u, v)=0$ for every $v \in \mathcal{F} \cap C_{c}(D)$.

If one assumes a priori that $u \in \mathcal{F}$, then the equivalence of (a) and (b) is easy to establish. See Remarks 2.8 (i) and 2.10 below.

In next section, we give precise definitions and statements of the main results and their proofs. Three examples are given to illustrate the main results of this paper. Extensions to general symmetric right processes on Lusin spaces including infinite dimensional spaces are mentioned at the end of this paper. We use ":=" as a means of definition. For two real numbers $a$ and $b, a \wedge b:=\min \{a, b\}$.

\section{MAin Results}

Let $X=\left(\Omega, \mathcal{F}_{\infty}, \mathcal{F}_{t}, X_{t}, \zeta, \mathbf{P}_{x}, x \in E\right)$ be an $m$-symmetric Hunt process on a locally compact separable metric space $E$, where $m$ is a positive Radon measure on $E$ with full topological support. A cemetery state $\partial$ is added to $E$ to form $E_{\partial}:=$ $E \cup\{\partial\}$ as its one-point compactification, and $\Omega$ is the totality of right-continuous, left-limited sample paths from $\left[0, \infty\left[\right.\right.$ to $E_{\partial}$ that hold the value $\partial$ once attaining it. For any $\omega \in \Omega$, we set $X_{t}(\omega):=\omega(t)$. Let $\zeta(\omega):=\inf \left\{t \geq 0 \mid X_{t}(\omega)=\partial\right\}$ be the lifetime of $X$. As usual, $\mathcal{F}_{\infty}$ and $\mathcal{F}_{t}$ are the minimal augmented $\sigma$-algebras obtained from $\mathcal{F}_{\infty}^{0}:=\sigma\left\{X_{s} \mid 0 \leq s<\infty\right\}$ and $\mathcal{F}_{t}^{0}:=\sigma\left\{X_{s} \mid 0 \leq s \leq t\right\}$ under $\left\{\mathbf{P}_{x}: x \in E\right\}$. For a Borel subset $B$ of $E, \tau_{B}:=\inf \left\{t>0 \mid X_{t} \notin B\right\}$ (the exit time of $B$ ) and $\sigma_{B}:=\inf \left\{t \geq 0 \mid X_{t} \in B\right\}$ (the entrance time of $B$ ) are $\left(\mathcal{F}_{t}\right.$ )-stopping times.

The transition semigroup $\left\{P_{t}: t \geq 0\right\}$ of $X$ is defined by

$$
P_{t} f(x):=\mathbf{E}_{x}\left[f\left(X_{t}\right)\right]=\mathbf{E}_{x}\left[f\left(X_{t}\right): t<\zeta\right], \quad t \geq 0 .
$$

Each $P_{t}$ may be viewed as an operator on $L^{2}(E, m)$, and taken as a whole, these operators form a strongly continuous semigroup of self-adjoint contractions. The Dirichlet form associated with $X$ is the bilinear form

$$
\mathcal{E}(u, v):=\lim _{t \downarrow 0} t^{-1}\left(u-P_{t} u, v\right)_{m}
$$

defined on the space

$$
\mathcal{F}:=\left\{u \in L^{2}(E ; m) \mid \sup _{t>0} t^{-1}\left(u-P_{t} u, u\right)_{m}<\infty\right\} .
$$


Here we use the notation $(f, g)_{m}:=\int_{E} f(x) g(x) m(d x)$. We assume that $(\mathcal{E}, \mathcal{F})$ is a regular Dirichlet form on $L^{2}(E ; m)$; that is, $C_{c}(E) \cap \mathcal{F}$ is dense both in $\left(C_{c}(E),\|\cdot\|_{\infty}\right)$ and in $\left(\mathcal{F}, \mathcal{E}_{1}\right)$. Here $C_{c}(E)$ is the space of continuous functions with compact support in $E$ and $\mathcal{E}_{1}(u, u):=\mathcal{E}(u, u)+(u, u)_{m}$. However to ensure a wide scope of applicability, we do not assume that the process $X$ (or equivalently, its associated Dirichlet form $(\mathcal{E}, \mathcal{F}))$ is $m$-irreducible.

We refer readers to [4] and 9] for the following known facts. The extended Dirichlet space $\mathcal{F}_{e}$ is the space of all functions $f$ on $E$ so that there is an $\mathcal{E}$-Cauchy sequence $\left\{f_{n}, n \geq 1\right\} \subset \mathcal{F}$ so that $f_{n}$ converges to $f m$-a.e. on $E$. For such an $f \in \mathcal{F}_{e}, \mathcal{E}(f, f):=\lim _{n \rightarrow \infty} \mathcal{E}\left(f_{n}, f_{n}\right)$. Every $f \in \mathcal{F}_{e}$ admits a quasi-continuous version (cf. [9, Theorem 2.1.7]). Throughout this paper, we always assume that every function in $\mathcal{F}_{e}$ is represented by its quasi-continuous version, which is unique up to a set of zero capacity (that is, quasi-everywhere, or q.e. for abbreviation). We adopt the convention that any function $f$ defined on $E$ is extended to $E_{\partial}$ by taking $f(\partial)=0$ and that $X_{\infty}(\omega):=\partial$ for every $\omega \in \Omega$. It is known that $\mathcal{F}_{e} \cap L^{2}(E ; m]=\mathcal{F}$. The extended Dirichlet form $\left(\mathcal{E}, \mathcal{F}_{e}\right)$ admits the following Beurling-Deny decomposition (cf. 4, Theorem 4.3.3] or [9, Theorem 5.3.1]):

$$
\mathcal{E}(u, u)=\mathcal{E}^{(c)}(u, u)+\frac{1}{2} \int_{E \times E}(u(x)-u(y))^{2} J(d x, d y)+\int_{E} u(x)^{2} \kappa(d x),
$$

where $\mathcal{E}^{(c)}$ is the strongly local part of $(\mathcal{E}, \mathcal{F}), J$ the jumping measure and $\kappa$ the killing measure of $(\mathcal{E}, \mathcal{F})$ (or of $X$ ). For $u, v \in \mathcal{F}_{e}, \mathcal{E}^{(c)}(u, v)$ can also be expressed by the mutual energy measure $\frac{1}{2} \mu_{\langle u, v\rangle}^{c}(E)$, which is the signed Revuz measure associated with $\frac{1}{2}\left\langle M^{u, c}, M^{v, c}\right\rangle$. Here for $u \in \mathcal{F}_{e}, M^{u, c}$ denotes the continuous martingale part of the square integrable martingale additive functional $M^{u}$ of $X$ in the Fukushima's decomposition (cf. [9, Theorem 5.2.2]) of

$$
u\left(X_{t}\right)-u\left(X_{0}\right)=M_{t}^{u}+N_{t}^{u}, \quad t \geq 0
$$

where $N^{u}$ is a continuous additive functional of $X$ having zero energy. When $u=v$, it is customary to write $\mu_{\langle u, u\rangle}^{c}$ as $\mu_{\langle u\rangle}^{c}$. The measure $\mu_{\langle u, v\rangle}^{c}$ enjoys the strong local property in the sense that if $u \in \mathcal{F}_{e}$ is constant on a nearly Borel quasi-open set $D$, then $\mu_{\langle u, v\rangle}^{c}(D)=0$ for every $v \in \mathcal{F}_{e}$ (see [4, Proposition 4.3.1]). For $u \in \mathcal{F}$, let $\mu_{\langle u\rangle}$ be the Revuz measure of $\left\langle M^{u}\right\rangle$. Then it holds that

$$
\mathcal{E}(u, u)=\frac{1}{2} \mu_{\langle u\rangle}(E)+\frac{1}{2} \int_{E} u(x)^{2} \kappa(d x) .
$$

For an open subset $D$ of $E$, we use $X^{D}$ to denote the subprocess of $X$ killed upon leaving $D$. The Dirichlet form of $X^{D}$ on $L^{2}(D ; m)$ is $\left(\mathcal{E}, \mathcal{F}^{D}\right)$, where $\mathcal{F}^{D}:=\{u \in$ $\mathcal{F} \mid u=0$ q.e. on $\left.D^{c}\right\}$. It is known (cf. [4, Theorem 3.3.9] or [9, Theorem 4.4.3]) that $\left(\mathcal{E}, \mathcal{F}^{D}\right)$ is a regular Dirichlet form on $L^{2}(D ; m)$. Let $\mathcal{F}_{e}^{D}:=\left\{u \in \mathcal{F}_{e} \mid u=\right.$ 0 q.e. on $\left.D^{c}\right\}$. Then $\mathcal{F}_{e}^{D}$ is the extended Dirichlet space of $\left(\mathcal{E}, \mathcal{F}^{D}\right)$ (see Theorem 3.4.9 of [4]). A function $f$ is said to be locally in $\mathcal{F}^{D}$, denoted as $f \in \mathcal{F}_{\text {loc }}^{D}$, if for every relatively compact subset $U$ of $D$, there is a function $g \in \mathcal{F}^{D}$ such that $f=g m$-a.e. on $U$. Every $f \in \mathcal{F}_{\text {loc }}^{D}$ admits an $m$-version that is quasi-continuous on $D$. Throughout this paper, we always assume that every function in $\mathcal{F}_{\text {loc }}^{D}$ when restricted to $D$ is represented by its quasi-continuous version. By the strong local property of $\mu_{\langle u, v\rangle}^{c}$ for $u, v \in \mathcal{F}, \mu_{\langle u, v\rangle}^{c}$ is well defined on $D$ for every $u, v \in \mathcal{F}_{\text {loc }}^{D}$. We use $L_{\text {loc }}^{\infty}(D ; m)$ to denote the $m$-equivalent class of locally bounded functions on $D$. 
Let $(N(x, d y), H)$ be a Lévy system of $X$ (cf. [3] or [9]). Then

$$
J(d x, d y)=N(x, d y) \mu_{H}(d x) \quad \text { and } \quad \kappa(d x):=N(x, \partial) \mu_{H}(d x),
$$

where $\mu_{H}$ is the Revuz measure of the positive continuous additive functional $H$ of $X$.

Definition 2.1. Let $D$ be an open subset of $E$. We say a function $u$ is harmonic in $D$ (with respect to the process $X$ ) if for every relatively compact open subset $U$ of $D, t \mapsto u\left(X_{t \wedge \tau_{U}}\right)$ is a uniformly integrable $\mathbf{P}_{x}$-martingale for q.e. $x \in U$.

To derive an analytic characterization of harmonic functions in $D$ in terms of an extension of quadratic form $(\mathcal{E}, \mathcal{F})$, we need some preparation. Let $r_{t}$ denote the time-reversal operator defined on the path space $\Omega$ of $X$ as follows: For $\omega \in\{t<\zeta\}$,

$$
r_{t}(\omega)(s)= \begin{cases}\omega((t-s)-) & \text { if } 0 \leq s<t \\ \omega(0) & \text { if } s \geq t .\end{cases}
$$

(It should be borne in mind that the restriction of the measure $\mathbf{P}_{m}$ to $\mathcal{F}_{t}$ is invariant under $r_{t}$ on $\Omega \cap\{\zeta>t\}$.)

Lemma 2.2. If $u \in \mathcal{F}_{e}$ has $\mathcal{E}(u, u)=0$, then

$$
\mathbf{P}_{x}\left(u\left(X_{t}\right)=u\left(X_{0}\right) \text { for every } t \geq 0\right)=1 \quad \text { for q.e. } x \in E .
$$

In other words, for q.e. $x \in E, E_{x}:=\{y \in E: u(y)=u(x)\}$ is an invariant set with respect to the process $X$ in the sense that $\mathbf{P}_{x}\left(X[0, \infty) \subset E_{x}\right)=1$. This in particular implies that if, in addition, $\mathbf{P}_{x}(\zeta<\infty)>0$ for q.e. $x \in E$, then $u=0$ q.e. on $E$.

Proof. It is known (see, e.g., [4, Theorem 6.6.2]) that the following Lyons-Zheng forward-backward martingale decomposition holds for $u \in \mathcal{F}_{e}$ :

$$
u\left(X_{t}\right)-u\left(X_{0}\right)=\frac{1}{2} M_{t}^{u}-\frac{1}{2} M_{t}^{u} \circ r_{t} \quad \mathbf{P}_{m} \text {-a.e. on }\{t<\zeta\} .
$$

As $\mu_{\langle u\rangle}(E) \leq 2 \mathcal{E}(u, u)=0$, we have $M^{u}=0$, and so $u\left(X_{t}\right)=u\left(X_{0}\right) \mathbf{P}_{m^{-}}$-a.s. on $\{t<\zeta\}$ for every $t>0$. This implies via Fukushima's decomposition that $N^{u}=0$ on $[0, \zeta)$ and hence on $[0, \infty) \mathbf{P}_{m}$-a.s. Consequently, $\mathbf{P}_{x}\left(u\left(X_{t}\right)-u\left(X_{0}\right)=M_{t}^{u}+N_{t}^{u}=0\right.$ for every $t \geq 0)=1$ for q.e. $x \in E$. This proves the lemma.

Since $(\mathcal{E}, \mathcal{F})$ is a regular Dirichlet form on $L^{2}(E ; m)$, for any relatively compact open sets $U, V$ with $\bar{U} \subset V$, there is $\phi \in \mathcal{F} \cap C_{c}(E)$ so that $\phi=1$ on $U$ and $\phi=0$ on $V^{c}$. Consequently,

$$
J\left(U, V^{c}\right)=\int_{U \times V^{c}}(\phi(x)-\phi(y))^{2} J(d x, d y) \leq 2 \mathcal{E}(\phi, \phi)<\infty .
$$

For an open set $D \subset E$, consider the following two conditions for the function $u$ on $E$. For any relatively compact open sets $U, V$ with $\bar{U} \subset V \subset \bar{V} \subset D$,

$$
\int_{U \times(E \backslash V)}|u(y)| J(d x, d y)<\infty
$$

and

$$
\mathbf{1}_{U}(x) \mathbf{E}_{x}\left[\left(\left(1-\phi_{V}\right)|u|\right)\left(X_{\tau_{U}}\right)\right] \in \mathcal{F}_{e}^{U},
$$

where $\phi_{V} \in C_{c}(D) \cap \mathcal{F}$ with $0 \leq \phi_{V} \leq 1$ and $\phi_{V}=1$ on $V$. Note that both conditions (2.4) and (2.5) are automatically satisfied when $X$ is a diffusion, since 
in this case the jumping measure $J$ vanishes and $X_{\tau_{U}} \in \partial U$ on $\left\{\tau_{U}<\zeta\right\}$. In view of (2.3), every bounded function $u$ satisfies condition (2.4). In fact by the following lemma, every bounded function $u$ also satisfies condition (2.5).

Lemma 2.3. Suppose that $u$ is a function on $E$ satisfying condition (2.4) and that for any relatively compact open sets $U, V$ with $\bar{U} \subset V \subset \bar{V} \subset D$,

$$
\sup _{x \in U} \mathbf{E}_{x}\left[\left(\mathbf{1}_{V^{c}}|u|\right)\left(X_{\tau_{U}}\right)\right]<\infty .
$$

Then (2.5) holds for $u$.

In many concrete cases such as in Examples 2.12 2.14 below, one can show that condition (2.4) implies condition (2.6). To prove the above lemma, we need the following result. Observe that the process $X$ is not assumed to be transient.

Lemma 2.4. Suppose that $\nu$ is a smooth measure on $E$ whose corresponding positive continuous additive functional (PCAF) of $X$ is denoted as $A^{\nu}$. Define $G \nu(x):=\mathbf{E}_{x}\left[A_{\zeta}^{\nu}\right]$. If $\int_{E} G \nu(x) \nu(d x)<\infty$, then $G \nu \in \mathcal{F}_{e}$. Moreover,

$$
\mathcal{E}(G \nu, u)=\int_{E} u(x) \nu(d x) \quad \text { for every } u \in \mathcal{F}_{e} .
$$

Proof. First assume that $m(E)<\infty$. It is easy to check directly that $\{x \in E$ : $\left.\mathbf{E}_{x}\left[A_{\zeta}^{\nu}\right]>j\right\}$ is finely open for every integer $j \geq 1$. So $K_{j}:=\{G \nu \leq j\}$ is finely closed. Since $G \nu<\infty \nu$-a.e. on $E$, we have $\nu\left(E \backslash \bigcup_{j=1}^{\infty} K_{j}\right)=0$. Define $\nu_{j}:=\mathbf{1}_{K_{j}} \nu$. Clearly for $x \in K_{j}, G \nu_{j}(x) \leq G \nu(x) \leq j$, while for $x \in K_{j}^{c}$,

$$
G \nu_{j}(x)=\mathbf{E}_{x}\left[\int_{0}^{\zeta} \mathbf{1}_{K_{j}}\left(X_{s}\right) d A_{s}^{\nu}\right]=\mathbf{E}_{x}\left[G \nu_{j}\left(X_{\sigma_{K_{j}}}\right)\right] \leq j .
$$

So $f_{j}:=G \nu_{j} \leq j$ on $E$ and hence is in $L^{2}(E ; m)$. Since by [4, Theorem 4.1.1] or [9, Theorem 5.1.3]

$\lim _{t \rightarrow 0} \frac{1}{t}\left(f_{j}-P_{t} f_{j}, f_{j}\right)_{m}=\lim _{t \rightarrow 0} \frac{1}{t} \mathbf{E}_{f_{j} \cdot m}\left[A_{t}^{\nu_{j}}\right]=\int_{E} f_{j}(x) \nu_{j}(d x) \leq \int_{E} G \nu(x) \nu(d x)<\infty$,

we have $f_{j} \in \mathcal{F}$ with $\mathcal{E}\left(f_{j}, f_{j}\right) \leq \int_{E} G \nu(x) \nu(d x)$. The same calculation shows that for $i>j, f_{i}-f_{j}=\mathbf{E}_{x}\left[A_{\zeta}^{\mathbf{1}_{K_{i} \backslash K_{j}} \cdot \nu}\right]$ and

$$
\mathcal{E}\left(f_{i}-f_{j}, f_{i}-f_{j}\right)=\int_{K_{i} \backslash K_{j}}\left(f_{i}-f_{j}\right)(x) \nu(d x) \leq \int_{K_{l} \backslash K_{j}} G \nu(x) \nu(d x),
$$

which tends to zero as $i, j \rightarrow \infty$; that is, $\left\{f_{j}, j \geq 1\right\}$ is an $\mathcal{E}$-Cauchy sequence in $\mathcal{F}$. As $\lim _{j \rightarrow \infty} f_{j}=f$ on $E$, we conclude that $f \in \mathcal{F}_{e}$. We deduce from (2.8) that

$$
\mathcal{E}(f, f)=\lim _{j \rightarrow \infty} \mathcal{E}\left(f_{j}, f_{j}\right)=\int_{E} G \nu(x) \nu(d x)
$$

Moreover, for $u \in \mathcal{F}_{b}^{+}$, by [4, Theorem 4.1.1] (or [9, Theorem 5.1.3]) and the dominated convergence theorem, we have

$$
\begin{aligned}
\mathcal{E}(G \nu, u) & =\lim _{j \rightarrow \infty} \mathcal{E}\left(f_{j}, u\right)=\lim _{j \rightarrow \infty} \lim _{t \rightarrow 0} \frac{1}{t}\left(f_{j}-P_{t} f_{j}, u\right) \\
& =\lim _{j \rightarrow \infty} \int_{E} u(x) \mathbf{1}_{K_{j}}(x) \nu(d x)=\int_{E} u(x) \nu(d x) .
\end{aligned}
$$


Since the linear span of $\mathcal{F}_{b}^{+}$is $\mathcal{E}$-dense in $\mathcal{F}_{e}$, we have established (2.7).

For a general $\sigma$-finite measure $m$, take a strictly positive $m$-integrable Borel measurable function $g$ on $E$ and define $\mu=g \cdot m$. Then $\mu$ is a finite measure on $E$. Let $Y$ be the time-change of $X$ via measure $\mu$; that is, $Y_{t}=X_{\tau_{t}}$, where $\tau_{t}=\inf \left\{s>0: \int_{0}^{s} g\left(X_{s}\right) d s>t\right\}$. The time-changed process $Y$ is $\mu$-symmetric. Let $\left(\mathcal{E}^{Y}, \mathcal{F}^{Y}\right)$ be the Dirichlet form of $Y$ on $L^{2}(E ; \mu)$. Then it is known that $\mathcal{F}_{e}^{Y}=\mathcal{F}_{e}$ and $\mathcal{E}^{Y}=\mathcal{E}$ on $\mathcal{F}_{e}$ (see (5.2.17) of [4]). The measure $\nu$ is also a smooth measure with respect to the process $Y$. It is easy to verify that the PCAF $A^{Y, \nu}$ of $Y$ corresponding to $\nu$ is related to the corresponding PACF $A^{\nu}$ of $X$ by

$$
A_{t}^{Y, \nu}=A_{\tau_{t}}^{\nu} \quad \text { for } t \geq 0 .
$$

In particular, we have $G^{Y} \nu(x)=G \nu$ on $E$. Since we just proved that the lemma holds for $Y$, we conclude that the lemma also holds for $X$.

Proof of Lemma 2.3. For relatively compact open sets $U, V$ with $\bar{U} \subset V \subset \bar{V} \subset$ $D$ and $\phi_{V} \in \mathcal{F} \cap C_{c}(D)$ with $0 \leq \phi_{V} \leq 1$ and $\phi_{V}=1$ on $V$, let $f(x):=$ $\mathbf{1}_{U}(x) \mathbf{E}_{x}\left[\left(\left(1-\phi_{V}\right)|u|\right)\left(X_{\tau_{U}}\right)\right]$, which is bounded by condition (2.6). Note that $1-\phi_{V}=0$ on $V$. Using the Lévy system of $X$, we have

$$
f(x)=\mathbf{E}_{x}\left[\int_{0}^{\tau_{U}}\left(\int_{E \backslash V}\left(1-\phi_{V}\left(X_{s}\right)\right)|u|\left(X_{s}\right) N\left(X_{s}, d y\right)\right) d H_{s}\right] \quad \text { for } x \in E .
$$

Note that the Revuz measure for PCAF

$$
t \mapsto \int_{0}^{t \wedge \tau_{U}}\left(\int_{E \backslash V}\left(1-\phi_{V}\left(X_{s}\right)\right)|u|(y) N\left(X_{s}, d y\right)\right) d H_{s}
$$

of $X^{U}$ is $\mu:=\left(\int_{E \backslash V}\left(1-\phi_{V}(x)\right)|u|(x) N(x, d y)\right) d \mu_{H}$, and so $f=G_{U} \mu$. Since by condition (2.4)

$$
\begin{aligned}
\mu(U) & =\int_{U}\left(\int_{E \backslash V}\left(1-\phi_{V}(y)\right)|u(y)| N(x, d y)\right) \mu_{H}(d x) \\
& \leq \int_{U}\left(\int_{E \backslash V}|u(y)| N(x, d y)\right) \mu_{H}(d x)<\infty,
\end{aligned}
$$

we have $\int_{U} G_{U} \mu(x) \mu(d x) \leq\|f\|_{\infty} \mu(U)<\infty$. Applying Lemma 2.4 to $X^{U}$ yields that $f \in \mathcal{F}_{e}^{U}$.

Lemma 2.5. Let $D$ be an open subset of E. Every $u \in \mathcal{F}_{e}$ that is locally bounded on $D$ satisfies conditions (2.4) and (2.5).

Proof. Let $u \in \mathcal{F}_{e}$ be locally bounded on $D$. For any relatively compact open sets $U, V$ with $\bar{U} \subset V \subset \bar{V} \subset D$, take $\phi \in \mathcal{F} \cap C_{c}(D)$ such that $\phi=1$ on $U$ and $\phi=0$ on $V^{c}$. Then $u \phi \in \mathcal{F}_{e}$ and

$$
\begin{aligned}
\int_{U \times(E \backslash V)} u(y)^{2} J(d x, d y) & \left.=\int_{U \times(E \backslash V)}((1-\phi) u)(x)-((1-\phi) u)(y)\right)^{2} J(d x, d y) \\
& \leq 2 \mathcal{E}(u-u \phi, u-u \phi)<\infty .
\end{aligned}
$$

This together with (2.3) implies that

$$
\int_{U \times(E \backslash V)}|u(y)| J(d x, d y) \leq \frac{1}{2} \int_{U \times(E \backslash V)}\left(1+u(y)^{2}\right) J(d x, d y)<\infty .
$$


Let $\phi_{V} \in \mathcal{F} \cap C_{c}(D)$ be such that $0 \leq \phi_{V} \leq 1$ with $\phi_{V}=1$ on $V$. Note that $|u| \in \mathcal{F}_{e}$ is locally bounded on $D$, and so $\left(1-\phi_{V}\right)|u|=|u|-\phi_{V}|u| \in \mathcal{F}_{e}$. Thus it follows from [4, Theorem 3.4.8] or [9, Theorem 4.6.5] that

$$
\mathbf{1}_{U}(x) \mathbf{E}_{x}\left[\left(\left(1-\phi_{V}\right)|u|\right)\left(X_{\tau_{U}}\right)\right]=\mathbf{E}_{x}\left[\left(\left(1-\phi_{V}\right)|u|\right)\left(X_{\tau_{U}}\right)\right]-\left(1-\phi_{V}\right)|u| \in \mathcal{F}_{e}^{U} .
$$

Lemma 2.6. Let $D$ be a relatively compact open set of $E$. Suppose $u$ is a function in $\mathcal{F}_{\text {loc }}^{D}$ that is locally bounded on $D$ and satisfies condition (2.4). Then for every $v \in C_{c}(D) \cap \mathcal{F}$, the expression

$$
\frac{1}{2} \mu_{\langle u, v\rangle}^{c}(D)+\frac{1}{2} \int_{E \times E}(u(x)-u(y))(v(x)-v(y)) J(d x, d y)+\int_{D} u(x) v(x) \kappa(d x)
$$

is well defined and finite; it will still be denoted as $\mathcal{E}(u, v)$.

Proof. Clearly the first and third terms are well defined and finite. To see that the second term is also well defined, let $U$ be a relatively compact open subset of $D$ such that $\operatorname{supp}[v] \subset U$. Since $u \in \mathcal{F}_{\text {loc }}^{D}$, there is $f \in \mathcal{F}$ so that $u=f m$-a.e. and hence q.e. on $U$. Under condition (2.4),

$$
\begin{aligned}
& \int_{E \times E}|(u(x)-u(y))(v(x)-v(y))| J(d x, d y) \\
\leq & \int_{U \times U}|(u(x)-u(y))(v(x)-v(y))| J(d x, d y)+2 \int_{U \times(E \backslash U)}|u(x) v(x)| J(d x, d y) \\
& +2 \int_{U}|v(x)| \int_{E \backslash U}|u(y)| J(d x, d y) \\
\leq & \int_{U \times U}|(f(x)-f(y))(v(x)-v(y))| J(d x, d y)+2\|u v\|_{\infty} J\left(\operatorname{supp}[v], U^{c}\right) \\
& +2\|v\|_{\infty} \int_{\operatorname{supp}[v] \times(E \backslash U)}|u(y)| J(d x, d y) \\
< & \infty
\end{aligned}
$$

In the last inequality we used (2.3) and the fact that $f, v \in \mathcal{F}$. This proves the lemma.

Theorem 2.7. Let $D$ be an open subset of E. Suppose that $u \in \mathcal{F}_{\text {loc }}^{D}$ is locally bounded on $D$ satisfying conditions (2.4)-(2.5) and that

$$
\mathcal{E}(u, v)=0 \quad \text { for every } v \in C_{c}(D) \cap \mathcal{F} .
$$

Then $u$ is harmonic in $D$. If $U$ is a relatively compact open subset of $D$ so that $\mathbf{P}_{x}\left(\tau_{U}<\infty\right)>0$ for q.e. $x \in U$, then $u(x)=\mathbf{E}_{x}\left[u\left(X_{\tau_{U}}\right)\right]$ for q.e. $x \in U$.

Proof. Take $\phi \in C_{c}(D) \cap \mathcal{F}$ such that $0 \leq \phi \leq 1$ and $\phi=1$ in an open neighborhood $V$ of $\bar{U}$. Then $\phi u \in \mathcal{F}^{D}$. So by [4, Theorem 3.4.8] or [9, Theorem 4.6.5], $h_{1}(x):=$ $\mathbf{E}_{x}\left[(\phi u)\left(X_{\tau_{U}}\right)\right] \in \mathcal{F}_{e}$ and $\phi u-h_{1} \in \mathcal{F}_{e}^{U}$. Moreover

$$
\mathcal{E}\left(h_{1}, v\right)=0 \quad \text { for every } v \in \mathcal{F}_{e}^{U} .
$$


Let $h_{2}(x):=\mathbf{E}_{x}\left[((1-\phi) u)\left(X_{\tau_{U}}\right)\right]$, which is well defined by condition (2.5). Note that by the Lévy system of $X$,

$$
\begin{aligned}
f(x) & :=\mathbf{1}_{U}(x) \mathbf{E}_{x}\left[((1-\phi)|u|)\left(X_{\tau_{U}}\right)\right] \\
& =\mathbf{1}_{U}(x) \mathbf{E}_{x}\left[\int_{0}^{\tau_{U}}\left(\int_{E \backslash V}((1-\phi)|u|)(z) N\left(X_{s} . d z\right)\right) d H_{s}\right] .
\end{aligned}
$$

Define $\mu(d x):=\mathbf{1}_{D}(x)\left(\int_{E \backslash V}((1-\phi)|u|)(z) N\left(X_{s} . d z\right)\right) \mu_{H}(d x)$, which is a smooth measure of $X^{U}$. In the following, for a smooth measure $\nu$ of $X^{U}$, we will use $G_{U} \nu$ to denote $\mathbf{E}_{x}\left[A_{\tau_{U}}^{\nu}\right]$, where $A^{\nu}$ is the PCAF of $X^{U}$ with Revuz measure $\nu$. Using such a notation, $f=G_{U} \mu$. We claim that $\mathbf{1}_{U} h_{2} \in \mathcal{F}_{e}^{U}$ and, for $v \in \mathcal{F}_{e}^{U}$,

$$
\mathcal{E}\left(\mathbf{1}_{D} h_{2}, v\right)=\int_{E} v(x) \mathbf{1}_{U}(x)\left(\int_{E \backslash V}((1-\phi) u)(z) N\left(X_{s}, d z\right)\right) \mu_{H}(d x) .
$$

Define

$$
\begin{aligned}
& \mu_{1}(d x):=\mathbf{1}_{D}(x)\left(\int_{E \backslash V}\left((1-\phi) u^{+}\right)(z) N\left(X_{s} . d z\right)\right) \mu_{H}(d x), \\
& \mu_{2}(d x):=\mathbf{1}_{D}(x)\left(\int_{E \backslash V}\left((1-\phi) u^{-}\right)(z) N\left(X_{s} . d z\right)\right) \mu_{H}(d x) .
\end{aligned}
$$

Observe that

$$
\begin{array}{r}
G_{U} \mu_{1}(x)=\mathbf{E}_{x}\left[\left((1-\phi) u^{+}\right)\left(X_{\tau_{U}}\right)\right] \quad \text { and } \quad G_{U} \mu_{2}(x)=\mathbf{E}_{x}\left[\left((1-\phi) u^{-}\right)\left(X_{\tau_{U}}\right)\right] \\
\text { for } x \in U .
\end{array}
$$

Clearly $G_{U} \mu_{1} \leq G_{U} \mu$. For $j \geq 1$, let $F_{j}:=\left\{x \in U: G_{U} \mu_{1}(x) \leq j\right\}$, which is a finely closed subset of $U$. Define $\nu_{j}:=\mathbf{1}_{F_{j}} \mu_{1}$. Then for $x \in F_{j}, G_{U} \nu_{j}(x) \leq G_{U} \mu_{1}(x) \leq j$, while for $x \in U \backslash F_{j}$,

$$
G_{U} \nu_{j}(x)=\mathbf{E}_{x}\left[G_{U} \nu_{j}\left(X_{\sigma_{F_{j}}}\right)\right] \leq j .
$$

In other words, we have $G_{U} \nu_{j} \leq j \wedge G_{U} \mu_{1} \leq j \wedge f$. As both $G_{U} \nu_{j}$ and $j \wedge f$ are excessive functions of $X^{U}$ and $m(U)<\infty$, we have by [4, Theorem 1.1.5 and Lemma 1.2.3] that $\left\{G_{U} \nu_{j}, j \wedge G_{U} \mu\right\} \subset \mathcal{F}^{U}$ and

$$
\mathcal{E}\left(G_{U} \nu_{j}, G_{U} \nu_{j}\right) \leq \mathcal{E}(j \wedge f, j \wedge f) \leq \mathcal{E}(f, f)<\infty .
$$

Moreover, for each $j \geq 1$, we have by [4, Theorem 4.1.1] or [9, Theorem 5.1.3] that

$$
\begin{aligned}
\mathcal{E}\left(G_{U} \nu_{j}, G_{U} \nu_{j}\right) & =\lim _{t \rightarrow 0} \frac{1}{t} \int_{E} G_{U}\left(\nu_{j}(x)-P_{t}^{U} G_{U} \nu_{j}(x)\right) G_{U} \nu_{j}(x) m(d x) \\
& =\lim _{t \rightarrow 0} \frac{1}{t} \int_{E} \mathbf{E}_{x}\left[A_{t \wedge \tau_{U}}^{\nu_{j}}\right] G_{U} \nu_{j}(x) m(d x) \\
& =\int_{U} G_{U} \nu_{j}(x) \mathbf{1}_{F_{j}}(x) \mu_{1}(d x),
\end{aligned}
$$

which increases to $\int_{U} G_{U} \mu_{1}(x) \mu_{1}(d x)$. Consequently, $\int_{U} G_{U} \mu_{1}(x) \mu_{1}(d x) \leq \mathcal{E}(f, f)$ $<\infty$. So we have by Lemma 2.4 applied to $X^{U}$ that $G_{U} \mu_{1} \in \mathcal{F}_{e}^{U}$ with $\mathcal{E}\left(G_{U} \mu_{1}, v\right)$ $=\int_{U} v(x) \mu_{1}(d x)$ for every $v \in \mathcal{F}_{e}^{U}$. Similarly we have $G_{U} \mu_{2} \in \mathcal{F}_{e}^{U}$ with $\mathcal{E}\left(G_{U} \mu_{2}, v\right)$ $=\int_{U} v(x) \mu_{2}(d x)$ for every $v \in \mathcal{F}_{e}^{U}$. It follows that $\mathbf{1}_{U} h_{2}=G_{U} \mu_{1}-G_{U} \mu_{2} \in \mathcal{F}_{e}^{U}$, and claim (2.12) is established. 
As $h_{2}=\mathbf{1}_{U} h_{2}+(1-\phi) u$ and $(1-\phi) u$ satisfies condition (2.4), we have by Lemma 2.6 and (2.12) that for every $v \in C_{c}(U) \cap \mathcal{F}$,

$$
\begin{aligned}
\mathcal{E}\left(h_{2}, v\right)= & \mathcal{E}\left(1_{U} h_{2}, v\right)+\mathcal{E}((1-\phi) u, v) \\
= & \int_{E \times E} v(x)(1-\phi(y)) u(y) N(x, d y) \mu_{H}(d x) \\
& -\int_{E \times E} v(x)(1-\phi(y)) u(y) N(x, d y) \mu_{H}(d x) \\
= & 0 .
\end{aligned}
$$

This combined with (2.11) and condition (2.10) proves that

$$
\mathcal{E}\left(u-h_{1}-h_{2}, v\right)=0 \quad \text { for every } v \in C_{c}(U) \cap \mathcal{F} .
$$

Since $u-\left(h_{1}+h_{2}\right)=\left(\phi u-h_{1}\right)-\mathbf{1}_{D} h_{2} \in \mathcal{F}_{e}^{U}$ and $C_{c}(U) \cap \mathcal{F}$ is $\mathcal{E}$-dense in $\mathcal{F}_{e}^{U}$, the above display holds for every $v \in \mathcal{F}_{e}^{U}$. In particular, we have

$$
\mathcal{E}\left(u-h_{1}-h_{2}, u-h_{1}-h_{2}\right)=0 .
$$

By Lemma 2.2. $u\left(X_{t}\right)-h_{1}\left(X_{t}\right)-h_{2}\left(X_{t}\right)$ is a bounded $\mathbf{P}_{x}$-martingale for q.e. $x \in E$. As

$$
h_{1}(x)+h_{2}(x)=\mathbf{E}_{x}\left[u\left(X_{\tau_{U}}\right)\right] \quad \text { for } x \in U,
$$

the above implies that $t \mapsto u\left(X_{t \wedge \tau_{U}}\right)$ is a uniformly integrable $\mathbf{P}_{x}$-martingale for q.e. $x \in U$. If $\mathbf{P}_{x}\left(\tau_{U}<\infty\right)>0$ for q.e. $x \in U$, applying Lemma 2.2 to the Dirichlet form $\left(\mathcal{E}, \mathcal{F}^{U}\right)$, we have $u-h_{1}-h_{2}=0$ q.e. on $U$, and so $u(x)=\mathbf{E}_{x}\left[u\left(X_{\tau_{U}}\right)\right]$ for q.e. $x \in U$. This completes the proof of the theorem.

Remark 2.8. $\quad$ (i) The principal difficulty in the above proof is establishing (2.14) and that $u-\left(h_{1}+h_{2}\right) \in \mathcal{F}_{e}^{U}$ for general $u \in \mathcal{F}_{\text {loc }}^{D}$ satisfying conditions (2.4) and (2.5). If $u$ is assumed a priori to be in $\mathcal{F}_{e}$, these facts and therefore the theorem itself are then much easier to establish. Note that when $u \in \mathcal{F}_{e}$, it follows immediately from [4, Theorem 3.4.8] or [9, Theorem 4.6.5] that $h_{1}+h_{2}=\mathbf{E}_{x}\left[u\left(X_{\tau_{U}}\right)\right] \in \mathcal{F}_{e}$ enjoys property (2.14) and $u-\left(h_{1}+h_{2}\right) \in \mathcal{F}_{e}^{U}$. Therefore (2.15) holds, and consequently $u$ is harmonic in $D$.

(ii) If we assume that the process $X$ (or equivalently $(\mathcal{E}, \mathcal{F})$ ) is $m$-irreducible and that $U^{c}$ is not $m$-polar, then $\mathbf{P}_{x}\left(\tau_{U}<\infty\right)>0$ for q.e. $x \in U$ (cf. 4, Theorem 3.5.6] or [9]).

Theorem 2.9. Suppose $D$ is an open set of $E$ with $m(D)<\infty$ and $u$ is a function on $E$ satisfying condition (2.4) so that $u \in L^{\infty}(D ; m)$ and $\left\{u\left(X_{t \wedge \tau_{D}}\right), t \geq 0\right\}$ is a uniformly integrable $\mathbf{P}_{x}$-martingale for q.e. $x \in E$. Then

$$
u \in \mathcal{F}_{\text {loc }}^{D} \quad \text { and } \quad \mathcal{E}(u, v)=0 \quad \text { for every } v \in C_{c}(D) \cap \mathcal{F} .
$$

Proof. As for q.e. $x \in E,\left\{u\left(X_{t \wedge \tau_{D}}\right), t \geq 0\right\}$ is a uniformly integrable $\mathbf{P}_{x}$-martingale, $u\left(X_{t \wedge \tau_{D}}\right)$ converges in $L^{1}\left(\mathbf{P}_{x}\right)$ as well as $\mathbf{P}_{x}$-a.s. to some random variable $\xi$ as $t \rightarrow \infty$. By considering $\xi^{+}, \xi^{-}$and $u_{+}:=\mathbf{E}_{x}\left[\xi^{+}\right], u_{-}(x):=\mathbf{E}_{x}\left[\xi^{-}\right]$separately, we may and do assume without loss of generality that $u \geq 0$. Note that $\xi \mathbf{1}_{\left\{\tau_{D}<\infty\right\}}=$ $u\left(X_{\tau_{D}}\right)$. Define $u_{1}(x):=\mathbf{E}_{x}\left[u\left(X_{\tau_{D}}\right)\right]$ and $u_{2}(x):=\mathbf{E}_{x}\left[\xi \mathbf{1}_{\left\{\tau_{D}=\infty\right\}}\right]=u-u_{1}$.

Let $\left\{P_{t}^{D}, t \geq 0\right\}$ denote the transition semigroup of the subprocess $X^{D}$. Then for q.e. $x \in D$ and every $t>0$, by the Markov property of $X^{D}$,

$$
P_{t}^{D} u_{2}(x)=\mathbf{E}_{x}\left[u_{2}\left(X_{t}\right), t<\tau_{D}\right]=\mathbf{E}_{x}\left[\xi \mathbf{1}_{\left\{\tau_{D}=\infty\right\}} \cdot \theta_{t}, t<\tau_{D}\right]=u_{2}(x) .
$$


Since $u_{2} \in L^{2}(D ; m)$, by (2.1)-(2.2)

$$
u_{2} \in \mathcal{F}^{D} \quad \text { with } \quad \mathcal{E}\left(u_{2}, u_{2}\right)=0 .
$$

On the other hand,

$$
P_{t}^{D} u(x)=\mathbf{E}_{x}\left[u\left(X_{t}\right), t<\tau_{D}\right]=\mathbf{E}_{x}\left[u\left(X_{\tau_{D}}\right), t<\tau_{D}\right] \leq u(x) .
$$

Let $\left\{D_{n}, n \geq 1\right\}$ be an increasing sequence of relatively compact open subsets of $D$ with $\bigcup_{n \geq 1} D_{n}=D$ and define

$$
\sigma_{n}:=\inf \left\{t \geq 0: X_{t}^{D} \in D_{n}\right\} .
$$

Let $e_{n}(x)=\mathbf{E}_{x}\left[e^{-\sigma_{n}}\right], x \in D$, be the 1-equilibrium potential of $D_{n}$ with respect to the subprocess $X^{D}$. Clearly $e_{n} \in \mathcal{F}^{D}$ is 1-excessive with respect to the process $X^{D}, e_{n}(x)=1$ q.e. on $D_{n}$. Let $a:=\left\|\mathbf{1}_{D} u\right\|_{\infty}$. Then for every $t>0$,

$$
e^{-t} P_{t}^{D}\left(\left(a e_{n}\right) \wedge u\right)(x) \leq\left(\left(a e_{n}\right) \wedge u\right)(x) \quad \text { for q.e. } x \in D .
$$

By [4, Lemma 1.2.3] or [10, Lemma 8.7], we have $\left(a e_{n}\right) \wedge u \in \mathcal{F}^{D}$ for every $n \geq 1$. Since $\left(a e_{n}\right) \wedge u=u m$-a.e. on $D_{n}$, we have $u \in \mathcal{F}_{\text {loc }}^{D}$.

Let $U$ be a relatively compact open subset of $D$. Let $\phi \in C_{c}(D) \cap \mathcal{F}$ so that $0 \leq \phi \leq 1$ and $\phi=1$ in an open neighborhood $V$ of $\bar{U}$. Define for $x \in E$,

$$
h_{1}(x):=\mathbf{E}_{x}\left[(\phi u)\left(X_{\tau_{U}}\right)\right] \text { and } h_{2}(x):=\mathbf{E}_{x}\left[((1-\phi) u)\left(X_{\tau_{U}}\right)\right] .
$$

Then $u_{1}=h_{1}+h_{2}$ on $E$. Since $\phi u \in \mathcal{F}$, we know as in (2.11) that $h_{1} \in \mathcal{F}_{e}$ and

$$
\mathcal{E}\left(h_{1}, v\right)=0 \quad \text { for every } v \in \mathcal{F}_{e}^{U} .
$$

By the same argument as that for (2.13), we have

$$
\mathcal{E}\left(h_{2}, v\right)=0 \quad \text { for every } v \in \mathcal{F}_{e}^{U} .
$$

These together with (2.17) in particular imply that

$$
\mathcal{E}(u, v)=\mathcal{E}\left(h_{1}+h_{2}+u_{2}, v\right)=0 \quad \text { for every } v \in C_{c}(U) \cap \mathcal{F} .
$$

Since $U$ is an arbitrary relatively compact subset of $D$, we have

$$
\mathcal{E}(u, v)=0 \quad \text { for every } v \in C_{c}(D) \cap \mathcal{F} .
$$

This completes the proof.

Remark 2.10. As mentioned in the Introduction, the principal difficulty for the proof of the above theorem is establishing that a function $u$ harmonic in $D$ is in $\mathcal{F}_{\text {loc }}^{D}$ with $\mathcal{E}(u, v)=0$ for every $v \in \mathcal{F} \cap C_{c}(D)$. If a priori $u$ is assumed to be in $\mathcal{F}_{e}$, then Theorem[2.9 is easy to establish. In this case, it follows from [4, Theorem 3.4.8] or [9, Theorem 4.6.5] that $u_{1}=h_{1}+h_{2}=\mathbf{E}_{x}\left[u\left(X_{\tau_{U}}\right)\right] \in \mathcal{F}_{e}$ and that the second property of (2.14) holds. This together with (2.17) immediately implies that $u$ enjoys (2.16). (See also Proposition 2.5 of 1 for this simple case but under an additional assumption that $1 \in \mathcal{F}$ with $\mathcal{E}(1,1)=0$.)

Combining Theorems 2.7 and 2.9, we have the following.

Theorem 2.11. Let $D$ be an open subset of $E$. Suppose that $u$ is a function on $E$ that is locally bounded on $D$ and satisfies conditions (2.4) and (2.5). Then

(i) $u$ is harmonic in $D$ if and only if condition (2.16) holds. 
(ii) Assume that for every relatively compact open subset $U$ of $D, \mathbf{P}_{x}\left(\tau_{U}<\infty\right)$ $>0$ for q.e. $x \in U$. (By Remark 2.8 (ii), this condition is satisfied if $(\mathcal{E}, \mathcal{F})$ is $m$-irreducible.) Then $u$ is harmonic in $D$ if and only if for every relatively compact subset $U$ of $D, u\left(X_{\tau_{U}}\right) \in L^{1}\left(\mathbf{P}_{x}\right)$ and $u(x)=\mathbf{E}_{x}\left[u\left(X_{\tau_{U}}\right)\right]$ for q.e. $x \in U$.

Example 2.12 (Stable-like process on $\mathbb{R}^{d}$ ). Consider the following Dirichlet form $(\mathcal{E}, \mathcal{F})$ on $L^{2}\left(\mathbb{R}^{d}, d x\right)$, where

$$
\begin{aligned}
\mathcal{F}= & W^{\alpha / 2,2}\left(\mathbb{R}^{d}\right):=\left\{u \in L^{2}\left(\mathbb{R}^{d} ; d x\right):\right. \\
& \left.\int_{\mathbb{R}^{d} \times \mathbb{R}^{d}}(u(x)-u(y))^{2} \frac{1}{|x-y|^{d+\alpha}} d x d y<\infty\right\}, \\
\mathcal{E}(u, v)= & \frac{1}{2} \int_{\mathbb{R}^{d} \times \mathbb{R}^{d}}(u(x)-u(y))(v(x)-v(y)) \frac{c(x, y)}{|x-y|^{d+\alpha}} d x d y \quad \text { for } u, v \in \mathcal{F} .
\end{aligned}
$$

Here $d \geq 1, \alpha \in(0,2)$ and $c(x, y)$ is a symmetric function in $(x, y)$ that is bounded between two positive constants. In literature, $W^{\alpha, 2}\left(\mathbb{R}^{d}\right)$ is called the Sobolev space on $\mathbb{R}^{d}$ of fractional order $(\alpha / 2,2)$. For an open set $D \subset \mathbb{R}^{d}, W^{\alpha, 2}(D)$ is similarly defined as above but with $D$ in place of $\mathbb{R}^{d}$. It is easy to check that $(\mathcal{E}, \mathcal{F})$ is a regular Dirichlet form on $L^{2}\left(\mathbb{R}^{d} ; d x\right)$, and its associated symmetric Hunt process $X$ is called a symmetric $\alpha$-stable-like process on $\mathbb{R}^{d}$, which is studied in [5]. The process $X$ has strictly positive jointly continuous transition density function $p(t, x, y)$ and hence is irreducible. Moreover, there is a constant $c>0$ such that

$$
p(t, x, y) \leq c t^{-d / \alpha} \quad \text { for } t>0 \text { and } x, y \in \mathbb{R}^{d},
$$

and consequently by [8, Theorem 1],

$$
\sup _{x \in U} \mathbf{E}_{x}\left[\tau_{U}\right]<\infty
$$

for any open set $U$ having finite Lebesgue measure. When $c(x, y)$ is constant, the process $X$ is nothing but the rotationally symmetric $\alpha$-stable process on $\mathbb{R}^{d}$. In this example, the jumping measure

$$
J(d x, d y)=\frac{c(x, y)}{|x-y|^{d+\alpha}} d x d y .
$$

Hence for any non-empty open set $D \subset \mathbb{R}^{d}$, condition (2.4) is satisfied if and only if $\left(1 \wedge|x|^{-d-\alpha}\right) u(x) \in L^{1}\left(\mathbb{R}^{d}\right)$. Moreover, for such a function $u$ and relatively compact open sets $U, V$ with $\bar{U} \subset V \subset \bar{V} \subset D$, by the Lévy system of $X$,

$$
\begin{aligned}
\sup _{x \in U} \mathbf{E}_{x}\left[\left(\mathbf{1}_{V^{c}}|u|\right)\left(X_{\tau_{U}}\right)\right] & =\sup _{x \in U} \mathbf{E}_{x}\left[\int_{0}^{\tau_{U}}\left(\int_{V^{c}} \frac{c\left(X_{s}, y\right)\left|u\left(X_{s}\right)\right|}{\left|X_{s}-y\right|^{d+\alpha}} d y\right) d s\right] \\
& \leq\left(c \int_{\mathbb{R}^{d}}\left(1 \wedge|y|^{-d-\alpha}\right)|u(y)| d y\right) \sup _{x \in U} \mathbf{E}_{x}\left[\tau_{U}\right]<\infty .
\end{aligned}
$$

In other words, for this example, condition (2.6) and hence (2.5) is a consequence of (2.4). So Theorem 2.9 says that for an open set $D$ and a function $u$ on $\mathbb{R}^{d}$ that is locally bounded on $D$ with $\left(1 \wedge|x|^{-d-\alpha}\right) u(x) \in L^{1}\left(\mathbb{R}^{d}\right)$, the following are equivalent:

(i) $u$ is harmonic in $D$;

(ii) for every relatively compact subset $U$ of $D, u\left(X_{\tau_{U}}\right) \in L^{1}\left(\mathbf{P}_{x}\right)$ and $u(x)=$ $\mathbf{E}_{x}\left[u\left(X_{\tau_{U}}\right)\right]$ for q.e. $x \in U$; 
(iii) $u \in \mathcal{F}_{\text {loc }}^{D}=W_{\text {loc }}^{\alpha, 2}(D)$ and

$$
\int_{\mathbb{R}^{d} \times \mathbb{R}^{d}}(u(x)-u(y))(v(x)-v(y)) \frac{c(x, y)}{|x-y|^{d+\alpha}} d x d y=0
$$

for every $v \in C_{c}(D) \cap W^{\alpha / 2,2}\left(\mathbb{R}^{d}\right)$.

Example 2.13 (Diffusion process on a locally compact separable metric space). Let $(\mathcal{E}, \mathcal{F})$ be a local regular Dirichlet form on $L^{2}(E ; m)$, where $E$ is a locally compact separable metric space and $X$ is its associated Hunt process. In this case, $X$ has continuous sample paths, and so the jumping measure $J$ is null (cf. [9]). Hence conditions (2.4) and (2.5) are automatically satisfied. Let $D$ be an open subset of $E$ and $u$ be a function on $E$ that is locally bounded in $D$. Then by Theorem 2.11 $u$ is harmonic in $D$ if and only if condition (2.16) holds.

Now consider the following special case: $E=\mathbb{R}^{d}$ with $d \geq 1, m(d x)$ is the Lebesgue measure $d x$ on $\mathbb{R}^{d}, \mathcal{F}=W^{1,2}\left(\mathbb{R}^{d}\right):=\left\{u \in L^{2}\left(\mathbb{R}^{d} ; d x\right) \mid \nabla u \in L^{2}\left(\mathbb{R}^{d} ; d x\right)\right\}$ and

$$
\mathcal{E}(u, v)=\frac{1}{2} \sum_{i, j=1}^{d} \int_{\mathbb{R}^{d}} a_{i j}(x) \frac{\partial u(x)}{\partial x_{i}} \frac{\partial v(x)}{\partial x_{j}} d x \quad \text { for } u, v \in W^{1,2}\left(\mathbb{R}^{d}\right),
$$

where $\left(a_{i j}(x)\right)_{1 \leq i, j \leq d}$ is a $d \times d$-matrix valued measurable function on $\mathbb{R}^{d}$ that is uniformly elliptic and bounded. In literature, $W^{1,2}\left(\mathbb{R}^{d}\right)$ is the Sobolev space on $\mathbb{R}^{d}$ of order $(1,2)$. For an open set $D \subset \mathbb{R}^{d}, W^{1,2}(D)$ is similarly defined as above but with $D$ in place of $\mathbb{R}^{d}$. Then $(\mathcal{E}, \mathcal{F})$ is a regular local Dirichlet form on $L^{2}\left(\mathbb{R}^{d} ; d x\right)$, and its associated Hunt process $X$ is a conservative diffusion on $\mathbb{R}^{d}$ having jointly continuous transition density function. Let $D$ be an open set in $\mathbb{R}^{d}$. Then by Theorem 2.11 the following are equivalent for a locally bounded function $u$ on $D$ :

(i) $u$ is harmonic in $D$;

(ii) for every relatively compact open subset $U$ of $D, u\left(X_{\tau_{U}}\right) \in L^{1}\left(\mathbf{P}_{x}\right)$ and $u(x)=\mathbf{E}_{x}\left[u\left(X_{\tau_{U}}\right)\right]$ for q.e. $x \in U$;

(iii) $u \in W_{\text {loc }}^{1,2}(D)$ and $\sum_{i, j=1}^{d} \int_{\mathbb{R}^{d}} a_{i j}(x) \frac{\partial u(x)}{\partial x_{i}} \frac{\partial v(x)}{\partial x_{j}} d x=0$ for every $v \in C_{c}(D) \cap$ $W^{1,2}\left(\mathbb{R}^{d}\right)$.

In fact, in this case, it can be shown that every (locally bounded) harmonic function has a continuous version.

Example 2.14 (Diffusions with jumps on $\mathbb{R}^{d}$ ). Consider the following Dirichlet form $(\mathcal{E}, \mathcal{F})$, where $\mathcal{F}=W^{1,2}\left(\mathbb{R}^{d}\right)$ and

$$
\begin{aligned}
\mathcal{E}(u, v)= & \frac{1}{2} \sum_{i, j=1}^{d} \int_{\mathbb{R}^{d}} a_{i j}(x) \frac{\partial u(x)}{\partial x_{i}} \frac{\partial v(x)}{\partial x_{j}} d x \\
& +\frac{1}{2} \int_{\mathbb{R}^{d} \times \mathbb{R}^{d}}(u(x)-u(y))(v(x)-v(y)) \frac{c(x, y)}{|x-y|^{d+\alpha}} d x d y
\end{aligned}
$$

for $u, v \in W^{1,2}\left(\mathbb{R}^{d}\right)$. Here $d \geq 1,\left(a_{i j}(x)\right)_{1 \leq i, j \leq d}$ is a $d \times d$-matrix valued measurable function on $\mathbb{R}^{d}$ that is uniformly elliptic and bounded, $\alpha \in(0,2)$ and $c(x, y)$ is a symmetric function in $(x, y)$ that is bounded between two positive constants. It is easy to check that $(\mathcal{E}, \mathcal{F})$ is a regular Dirichlet form on $L^{2}\left(\mathbb{R}^{d} ; d x\right)$. Its associated 
symmetric Hunt process $X$ has both the diffusion and jumping components. Such a process has recently been studied in [6]. It is shown there that the process $X$ has a strictly positive jointly continuous transition density function $p(t, x, y)$ and hence is irreducible. Moreover, a sharp two-sided estimate is obtained in [6] for $p(t, x, y)$. In particular, there is a constant $c>0$ such that

$$
p(t, x, y) \leq c\left(t^{-d / \alpha} \wedge t^{-d / 2}\right) \quad \text { for } t>0 \text { and } x, y \in \mathbb{R}^{d} .
$$

Note that when $\left(a_{i j}\right)_{1 \leq i, j \leq d}$ is the identity matrix and $c(x, y)$ is constant, the process $X$ is nothing but the symmetric Lévy process that is the independent sum of a Brownian motion and a rotationally symmetric $\alpha$-stable process on $\mathbb{R}^{d}$. In this example, the jumping measure

$$
J(d x, d y)=\frac{c(x, y)}{|x-y|^{d+\alpha}} d x d y
$$

Hence for any non-empty open set $D \subset \mathbb{R}^{d}$, condition (2.4) is satisfied if and only if $\left(1 \wedge|x|^{-d-\alpha}\right) u(x) \in L^{1}\left(\mathbb{R}^{d}\right)$. By the same reasoning as that for (2.20), we see that for this example, condition (2.6) and hence (2.5) is implied by condition (2.4). So Theorem 2.9 says that for an open set $D$ and a function $u$ on $\mathbb{R}^{d}$ that is locally bounded on $D$ with $\left(1 \wedge|x|^{-d-\alpha}\right) u(x) \in L^{1}\left(\mathbb{R}^{d}\right)$, the following are equivalent:

(i) $u$ is harmonic in $D$ with respect to $X$;

(ii) for every relatively compact subset $U$ of $D, u\left(X_{\tau_{U}}\right) \in L^{1}\left(\mathbf{P}_{x}\right)$ and $u(x)=$ $\mathbf{E}_{x}\left[u\left(X_{\tau_{U}}\right)\right]$ for q.e. $x \in U$

(iii) $u \in W_{\mathrm{loc}}^{1,2}(D)$ such that for every $v \in C_{c}(D) \cap W^{1,2}\left(\mathbb{R}^{d}\right)$,

$$
\begin{aligned}
& \sum_{i, j=1}^{d} \int_{\mathbb{R}^{d}} a_{i j}(x) \frac{\partial u(x)}{\partial x_{i}} \frac{\partial v(x)}{\partial x_{j}} d x \\
& \quad+\int_{\mathbb{R}^{d} \times \mathbb{R}^{d}}(u(x)-u(y))(v(x)-v(y)) \frac{c(x, y)}{|x-y|^{d+\alpha}} d x d y=0
\end{aligned}
$$

Remark 2.15. It is possible to extend the results of this paper to a general $m$ symmetric right process $X$ on a Lusin space, where $m$ is a positive $\sigma$-finite measure with full topological support on $E$. In this case, the Dirichlet $(\mathcal{E}, \mathcal{F})$ of $X$ is a quasi-regular Dirichlet form on $L^{2}(E ; m)$. By [7], $(\mathcal{E}, \mathcal{F})$ is quasi-homeomorphic to a regular Dirichlet form on a locally compact separable metric space. So the results of this paper can be extended to the quasi-regular Dirichlet form setting by using this quasi-homeomorphism. However since the notion of an open set is not invariant under a quasi-homeomorphism, some modifications are needed. We need to replace the open set $D$ in Definition 2.1 by a quasi-open set $D$. Similar modifications are needed for conditions (2.4) and (2.5) as well. We say a function $u$ is harmonic in a quasi-open set $D \subset E$ if for every quasi-open subset $U \subset D$ with $\bar{U} \cap F_{k} \subset D$ for every $k \geq 1$, where $\left\{F_{k}, k \geq 1\right\}$ is an $\mathcal{E}$-nest consisting of compact sets, $t \mapsto u\left(X_{t \wedge \tau_{U \cap F_{k}}}\right)$ is a uniformly integrable $\mathbf{P}_{x}$-martingale for q.e. $x \in U \cap F_{k}$ 
and for every $k \geq 1$. The local Dirichlet space $\mathcal{F}_{\text {loc }}^{D}$ needs to be replaced by

$$
\begin{gathered}
\stackrel{\circ}{\mathcal{F}_{\text {loc }}^{D}}=\left\{u: \text { there is an increasing sequence of quasi-open sets }\left\{D_{n}\right\}\right. \\
\text { with } \bigcup_{n=1}^{\infty} D_{n}=D \text { q.e. and a sequence }\left\{u_{n}\right\} \subset \mathcal{F}^{D} \\
\text { such that } \left.u=u_{n} m \text {-a.e. on } D_{n}\right\} .
\end{gathered}
$$

Condition (2.16) should be replaced by

$$
\stackrel{\stackrel{\circ}{D}}{u} \quad \text { and } \quad \mathcal{E}(u, v)=0 \quad \text { for every } v \in \mathcal{F} \text { with } \mathcal{E} \text {-supp }[v] \subset D .
$$

Here $\mathcal{E}$-supp $[u]$ is the smallest quasi-closed set that $u$ vanishes $m$-a.e. on its complement. We leave the details to interested readers.

\section{ACKNOWLEDGEMENTS}

The author thanks Rich Bass and Takashi Kumagai for helpful discussions. He also thanks Rongchan Zhu for helpful comments.

\section{REFERENCES}

[1] M. T. Barlow, R. F. Bass, T. Kumagai and A. Teplyaev, Uniqueness of Brownian motion on Sierpinski carpets. Preprint, 2008.

[2] R. F. Bass, M. Kassmann and T. Kumagai, private communications, 2007.

[3] A. Benveniste and J. Jacod, Systèmes de Lévy des processus de Markov. Invent. Math. 21 (1973), 183-198. MR0343375 (49:8117)

[4] Z.-Q. Chen and M. Fukushima, Symmetric Markov Processes, Time Change and Boundary Theory. Book manuscript, 2009.

[5] Z.-Q. Chen and T. Kumagai, Heat kernel estimates for stable-like processes on $d$-sets. Stochastic Process Appl. 108 (2003), 27-62. MR2008600 (2005d:60135)

[6] Z.-Q. Chen and T. Kumagai, A priori Hölder estimate, parabolic Harnack principle and heat kernel estimates for diffusions with jumps. To appear in Rev. Mat. Iberoamericana.

[7] Z.-Q. Chen, Z.-M. Ma and M. Röckner, Quasi-homeomorphisms of Dirichlet forms, Nagoya Math. J. 136 (1994) 1-15. MR.1309378 (95m:31020)

[8] K. L. Chung, Greenian bounds for Markov processes. Potential Analysis 1 (1992), 83-92. MR.1245226(95d:60125)

[9] M. Fukushima, Y. Oshima and M. Takeda, Dirichlet Forms and Symmetric Markov Processes, Walter de Gruyter, Berlin-New York, 1994. MR1303354 (96f:60126)

[10] M. L. Silverstein, Symmetric Markov Processes. Lecture Notes in Math., 426, Springer-Verlag, Berlin-New York, 1974. MR0386032 (52:6891)

Department of Mathematics, University of Washington, Seattle, Washington 98195

E-mail address: zchen@math.washington.edu 